Editorial

\author{
Ana Luiza Gomes Pinto Navas ${ }^{1}$ \\ Larissa Berti2 \\ Emília Rodrigues Trindade ${ }^{3}$ \\ Pamela Papile Lunardel $0^{4}$
}

\section{Divulgação científica como forma de compartilhar conhecimento}

Em tempos em que a sociedade está cada vez mais conectada às mídias sociais, é necessário usá-las com uma ferramenta de comunicação e difusão do conhecimento, acessível e veloz. O uso das redes sociais como instrumento para disseminar o conhecimento gerado pela produção científica diminui a distância entre a pesquisa e a prática clínica, permitindo o acesso e o diálogo não somente de determinadas áreas do conhecimento, mas também a outros públicos. A potencial democratização do conhecimento técnicocientífico proporcionada pelo universo digital representou um rompimento de barreiras e, atualmente, uma fonte de agilidade na transmissão de conteúdos da ciência.

Tradicionalmente, a relevância de um periódico é mensurada por um indicador baseado em citações, seja no próprio periódico ou em outro, calculando-se o fator de impacto (FI). Há, no entanto, o reconhecimento do uso de métricas alternativas ou altmetrics (ALTernative article-level metrics), que foram adotadas no início da última década como referência às mensurações alternativas do impacto promovido por determinado produto científico $^{(1-3)}$. Obtém-se essa métrica por meio da atividade promovida por um artigo em plataformas digitais, monitorando-o por meio do seu número de identificação digital (DOI) e registrando a atenção direcionada a este nas mídias sociais ${ }^{(3)}$. Por mensurar uma dimensão distinta de impacto científico, as altmetrics permitem diversidade de alcance além do contexto acadêmico, com maior velocidade e abertura. Além disso, o retorno ao pesquisar sobre o alcance de seu artigo ocorre em menos tempo quando comparado ao cálculo do $\mathrm{FI}^{(1-3)}$. As altmetrics apresentam papéis e propósitos distintos dos métodos bibliométricos tradicionais, não os substituindo. A sua inclusão deve ser complementar, a fim de diminuir as limitações existentes, e compatível ao contexto atual de difusão do conhecimento em escala global ${ }^{(1-3)}$.

\footnotetext{
${ }^{1}$ Faculdade de Ciências Médicas da Santa Casa de São Paulo - São Paulo (SP), Brasil.

${ }^{2}$ Universidade Estadual Paulista "Júlio de Mesquita Filho" - UNESP - Marília (SP), Brasil.

${ }^{3}$ Universidade de Brasília - UNB - Brasília (DF) Brasil.

${ }^{4}$ Faculdade de Filosofia, Ciências e Letras de Ribeirão Preto da Universidade de São Paulo - FFCLRP - USP

- Ribeirão Preto (SP), Brasil.
}

Conflito de interesses: Nada a declarar.

Fontes de financiamento: Nada a declarar.
Endereço para correspondência: Ana Luiza Gomes Pinto Navas Buarque, São Paulo (SP), Brasil, CEP: 01221-020.

E-mail: analunavas@gmail.com 
Em sua página oficial, a Revista CoDAS apresenta a edição atual e todo o acervo do periódico. Também são divulgados nas contas do Twitter, Facebook e Instagram cujos números de alcance e interação são crescentes. Até o presente momento, foram realizadas 69 publicações referentes a artigos científicos da CoDAS e a divulgação dos mais recentes volumes e números disponibilizados. A rede social Instagram, com conta criada em 2018, apresenta 1.715 seguidores ativos com alcance médio de 1.900 contas e 2.600 impressões por publicação. Esse último índice indica o número de vezes que determinada publicação foi visualizada. A conta do Twitter, também criada em 2018, apresenta 57 seguidores e número médio de 230 impressões por publicação. Criada em 2016, a conta do Facebook apresenta 1.231 seguidores ativos com alcance médio por publicação de 200 contas.

O número de alcance e impressões decorre da interação dos leitores com as publicações, dos compartilhamentos e das hashtags \# relacionadas ao tema do artigo. No Instagram, a publicação que promoveu mais visualizações foi a do artigo de Luchesi et al. ${ }^{(4)}$, "Identificação das alterações de deglutição: percepção de pacientes com doenças neurodegenerativas". Nessa mesma conta, o artigo com mais interações em comentários e curtidas foi "Critérios para decanulação da traqueostomia: revisão de literatura"(5). Ainda, as publicações referentes à "Apraxia de fala e atraso de linguagem: a complexidade do diagnóstico e tratamento em quadros sintomáticos de crianças"(6) e "Hiperresponsividade auditiva no transtorno do espectro autista, terminologias e mecanismos fisiológicos envolvidos: revisão sistemática"(7) apresentaram números expressivos de visualização.

$\mathrm{Na}$ conta do Twitter, o maior número de engajamentos foi para a publicação de Casarin et al ${ }^{(8)}$ denominada "Bateria Montreal de Avaliação da Comunicação Breve - MAC B: fidedignidade e validade". No Facebook, os artigos com mais interação foram os de Pereira et al ${ }^{(9)}$ denominado "Associação entre hábitos orais deletérios e as estruturas e funções do sistema estomatognático: percepção dos responsáveis" e o de Silva et al. ${ }^{(10)}$, "Glendonald Auditory Screening Procedure (GASP): marcadores clínicos de desenvolvimento das habilidades de reconhecimento e compreensão auditiva em crianças usuárias de implante coclear".

Assim como nas redes sociais, outra mídia em que houve aumento de acesso foi a da Revista CoDAS. O artigo mais acessado nessa plataforma foi "Avaliação do Programa de Treinamento para memória de trabalho em idosos"(11), seguido de "Fadiga vocal em professores disfônicos que procuram atendimento fonoaudiológico"(12) e "Perfil miofuncional orofacial de pacientes com distúrbio do sono: relação com resultado da polissonografia" ${ }^{13}$, todos divulgados também nas redes sociais.

Quanto à interação por área da fonoaudiologia, ao analisar as três redes sociais da Revista CoDAS, o artigo que apresentou mais compartilhamentos na área da Voz foi o de Monteiro et al. ${ }^{(14)}$ denominado "Principais fatores que levam os professores de canto popular a buscar ajuda fonoaudiológica". Para a área da Linguagem, o artigo de destaque foi "Estrutura e coerência da narrativa oral de crianças com transtorno do déficit de atenção e hiperatividade"(15). O artigo "Hiper-responsividade auditiva no transtorno do espectro autista, terminologias e mecanismos fisiológicos envolvidos: revisão sistemática" ${ }^{(7)}$ apresentou mais compartilhamentos na área da Audiologia. Para a área da Disfagia, destacou-se o artigo de Freitas e Cardoso ${ }^{(16)}$ intitulado "Sintomas de disfagia em crianças com fissura labial e/ou palatina pré e pós-correção cirúrgica". O artigo mais compartilhado na área da Motricidade Orofacial foi "Características orofaciais de idosos funcionalmente independentes”(17). Nas áreas da Saúde Coletiva, Fluência e Gerontologia, os artigos que se destacaram em compartilhamento foram "Avaliação da assistência fonoaudiológica na estratégia de saúde da família pela perspectiva do usuário"(18), "Comparação da performance de fala em indivíduos gagos e fluentes"(19) e "Avaliação do Programa de Treinamento para memória de trabalho em idosos"(11), respectivamente.

A repercussão do trabalho da equipe de divulgação da CoDAS realizado até o presente momento permite afirmar que as mídias sociais são eficazes na ampla propagação da produção científica e permitem um diálogo mais próximo e ágil com a comunidade profissional e científica. Esses dados versam quanto a imprescindível continuidade dessa ação, contemplando diferentes temas, áreas, autores e instituições, contribuindo na difusão da ciência de qualidade.

\section{REFERÊNCIAS:}

1. Barros M. Altmetrics: métricas alternativas de impacto científico com base em redes sociais. Perspect. ciênc. inf. [online]. 2015; (20)2:19-37. http:// dx.doi.org/10.1590/1981-5344/1782.

2. Warren HR, Raison N, Dasgupta P. The rise of altmetrics. JAMA. 2017;317(2):131-2. http://dx.doi.org/10.1001/jama.2016.18346.

3. Taberner R. Altmetrics: Beyond the Impact Factor. Actas Dermosifiliogr 2018;109(2):95-7.

4. Luchesi KF, Campos BM, Mituuti CT. Identification of swallowing disorders: the perception of patients with neurodegenerative diseases. CoDAS. 2018;30(6): e20180027. https://doi.org/10.1590/2317-1782/20182018027.

5. Medeiros GC, Sassi FC, Lirani-Silva C, Andrade CRF. Criteria for tracheostomy decannulation: literature review. CoDAS. 2019;31(6):e20180228. https:// doi.org/10.1590/2317-1782/20192018228.

6. Catrini M, Lier-Devitto MF. Apraxia of speech and language delay: the complexity of diagnosis and treatment of symptomatic children. CoDAS 2019;31(5):e20180121. https://doi.org/10.1590/2317-1782/20192018121.

7. Stefanelli ACGF, Zanchetta S, Furtado EF. Auditory hyper-responsiveness in autism spectrum disorder, terminologies and physiological mechanisms involved: systematic review. CoDAS. 2020;32(3):e20180287. https://doi. org/10.1590/2317-1782/20192018287.

8. Casarin FS, Pagliarin KC, Altmann RF, Parente MAMP, Ferré P, Côté H et al. Montreal Communication Evaluation Brief Battery - MEC B: reliability and validity. CoDAS. 2020;32(1):e20180306. https://doi.org/10.1590/2317$1782 / 20192018306$.

9. Pereira TS, Oliveira F, Cardoso MCAF. Association between harmful oral habits and the structures and functions of the stomatognathic system: perception of parents/guardians. CoDAS. 2017;29(3):e20150301. https:// doi.org/10.1590/2317-1782/20172015301.

10. Silva BCS, Moret ALM, Silva LTN, Costa OA, Alvarenga KF, Silva-Comerlatto MP. Glendonald Auditory Screening Procedure (GASP): clinical markers of the development of auditory recognition and comprehension abilities in children using cochlear implants. CoDAS. 2019;31(4):e20180142. https:// doi.org/10.1590/2317-1782/20192018142.

11. Brito VV, Manhaes AG, Franca AI, Marins M. Evaluation of the Working Memory Training Program for the Elderly. CoDAS. 2019;31(3):e20180089. http://dx.doi.org/10.1590/2317-1782/20182018089.

12. Abou-Rafée M, Zambon F, Badaro F, Behlau M. Vocal fatigue in dysphonic teachers who seek treatment. CoDAS. 2019;31(3):e20180120. https://doi. org/10.1590/2317-1782/20182018120. 
13. Miranda VSG, Buffon G, Vidor DCGM. Orofacial myofunctional profile of patients with sleep disorders: relationship with result of polysomnography. CoDAS. 2019;31(3):e20180183. https://doi.org/10.1590/2317-1782/20182018183.

14. Monteiro JC, Madazio G, Pacheco C, Behlau M. Main reasons that lead popular music singing teachers to seek speech-language pathology assistance for their students. CoDAS. 2020;32(2):e20180242. https://doi. org/10.1590/2317-1782/20192018242.

15. Zenaro MP, Rossi NF, Souza ALDM, Giacheti CM. Oral narrative structure and coherence of children with attention deficit hyperactivity disorder. CoDAS. 2019;31(6):e20180197. https://doi.org/10.1590/2317-1782/20192018197.

16. Freitas JS, Cardoso MCAF. Symptoms of dysphagia in children with cleft lip and/or palate pre- and post-surgical correction. CoDAS. 2018;30(1):e20170018. https://doi.org/10.1590/2317-1782/20182017018.

17. Silva DNM, Couto EAB, Becker HMG, Bicalho MAC. Orofacial characteristics of functionally independent elders. CoDAS. 2017;29(4):e20160240. https:// doi.org/10.1590/2317-1782/20172016240.
18. Zanin LE, Albuquerque IMN, Carneiro MSM, Melo DH. Evaluation of speech language pathology care in the family health strategy from user perspective. CoDAS. 2017;29(6):e20160192. https://doi.org/10.1590/2317$1782 / 20172016192$.

19. Costa JB, Ritto AP, Juste FS, Andrade CRF. Comparison between the speech performance of fluent speakers and individuals who stutter. CoDAS. 2017;29(2):e20160136. https://doi.org/10.1590/2317$1782 / 20172016136$.

\section{Contribuição dos autores}

$A L G P N$ e $L B$ responsáveis pela idealização, redação e aprovação final do manuscrito; ERT e PPL responsáveis pelo levantamento e análise de dados, $e$ pela preparação e redação do manuscrito. 